\title{
Analysis of Snow Water Equivalent (SWE) of Snowpack by an Ultra Wide Band Step Frequency Continuous Wave Radar (SFCW)
}

\author{
Rafael Alonsoํㅜ José María García del Pozo ${ }^{2}$, Ismael Peruga ${ }^{3}$, Samuel Buisán ${ }^{4}$, José Adolfo Álvarez ${ }^{5}$ \\ ${ }^{1}$ Department of Applied Physics. University of Zaragoza, Zaragoza, Spain, ralonso@unizar.es \\ 2Department of Applied Physics. University of Zaragoza, Zaragoza, Spain, chgarcia@unizar.es \\ ${ }^{3}$ Department of Applied Physics. University of Zaragoza, Zaragoza, Spain, ismaperuga@gmail.com \\ ${ }^{4}$ Territorial Delegation of AEMET (Spanish State Meteorological Agency) in Aragón, Zaragoza, Spain, sbuisans@aemet.es \\ ${ }^{5}$ Ebro River Basin Authority (CHE), Zaragoza, Spain, jaalvarez@chebro.es
}

\begin{abstract}
A ground-based step frequency continuous wave radar (SFCW), based in a software defined radio (SDR), in the range from $150 \mathrm{MHz}$ to $6 \mathrm{GHz}$ has been designed, fabricated and tested under real conditions. The radar has been applied to measure the snow water equivalent (SWE) of snowpack in the Spanish Pyrenees. A matrix method is applied to solve the electromagnetic reflectance of multilayer cover snow including frequency and wetness dependence of complex relative dielectric permittivity of snow layers. An approximated method to obtain SWE is presented. The method is based on the comparison of measured reflected signal vs distance plot with the calculated with an "equivalent" snow layer. Preliminary results are presented and compared with those provided by a cosmic-ray neutron SWE gauge over the 2019 winter. These results suggest the viability of the proposed method.
\end{abstract}

Index Terms-snow, snow water equivalent, SWE, step frequency continous wave radar, SFCW, software defined radio, SDR, snowpack multilayer reflectance.

\section{INTRODUCTION}

\section{A. The Snow Water Equivalent Importance}

Snow is a great contribution to the water balance, climate and economy of many regions. The seasonal accumulation of snow acts as a form of natural regulation of great importance in the hydrological cycle. The parameter to characterize available water from the snow cover is the well-known snow water equivalent (SWE). SWE is the equivalent depth of water available if the snowpack melted into liquid water. This paper presents a near-surface-based technique for determining SWE from the measured complex spectral reflectance, in the range from $150 \mathrm{MHz}$ to $6 \mathrm{GHz}$, and the fit to an electromagnetic model for reflectance of the snowpack.

\section{B. Instrumentation and Techniques}

Ground-based remote sensing techniques for characterizing the physical properties of snow have been exhaustively discussed in the literature [1]. Conventional insitu techniques to non-destructively determine SWE are based on cosmic-ray neutron ( $\mathrm{CRN})$ or gamma radiation attenuation $[2,3]$ or acoustic signal delays [4], among others.

However, the most promising methods are those based on microwave radar analysis. In fact, over the past 40 years, numerous studies on radar applications to snow cover characterization have been conducted. Impulse waveform radars $[5,6]$ and FMCW radars [7-10] have been used to explore snowpack structure.

The recent advances in radar technology, propitiated by automotive radars or software defined radios (SDR), have resulted in low cost and small dimension equipment that will allow portable instrumentation with high vertical resolution to study properties such as depth, stratigraphy and SWE of the snow cover.

The aim of this paper is to show the operation of a stepped frequency continuous wave radar (SFCW), SDR based, and the application of an electromagnetic model of the multilayer snowpack to analyze the SWE of snow cover.

This paper is organized as follows. Section 2 presents the theory of a SFCW radar. Section 3 shows the electromagnetic model and the procedure to obtain SWE. Section 4 focuses on the description of the experimental set-up and the comparison between experimental and theoretical results. Finally, Section 5 summarizes the contributions of the present work.

\section{THEORY OF THE SFCW RADAR}

\section{A. Measurement Principle}

An SFCW [11] radar transmits consecutive trains of CW signals of increasing frequency towards targets. The received spectral in-phase $(I(f))$ and quadrature $(Q(f))$ signals reflected by targets are transformed into time-domain pulse signals using inverse Fourier transform (IFFT), which contain the transit time information or the "optical path" from different targets. Fig. 1 depicts the scheme of the system.

The SFCW radar has very narrow instantaneous bandwidth at each frequency due to the narrow pass filter width at the end of DC, $I$ and $Q$ signal processing, resulting in a high signal-to-noise ratio at the receiver. Its bandwidth, $B$, 
can be very wide, leading, according with general radar theory, to fine resolution, $\Delta R$, and given by:

$$
\Delta R=v / 2 B .
$$

The maximum unambiguous range, $R_{u}$, can be derived as:

$$
R_{u}=v / 2 \delta f,
$$

where $\delta f$ is the step frequency and $v$ the speed of wave. The main disadvantage of an SFCW radar is its long measurement time. Nevertheless, in our application, the snow cover is a "static" target and the measurement time is not relevant.

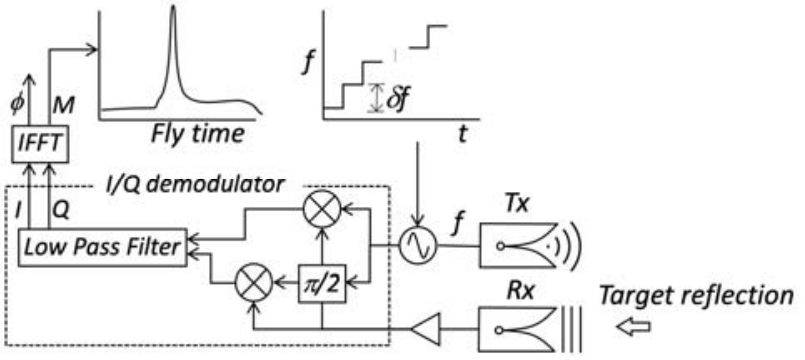

Fig. 1. SFCW system. The $T x$ oscillator generates trains of increasing frequency with $\delta f$ step. The reflected signal is demodulated $(I(f) / Q(f))$ and the inverse FFT of magnitude $(M(f))$ provides time information of the targets.

\section{SNOWPACK ELECTROMAGNETIC MODEL}

Many authors describe wave propagation in snow layered media with recursive relationships of transmitted and reflected rays [12]. However, we have used the $2 \times 2$ matrix method typically employed in planar multilayer optical structures and extensively described in the literature $[13,14]$.

\section{A. Matricial Multilayer Snowpack Electromagnetic Model}

The matrix formulation is an extremely useful form of the steady-state solution of Maxwell's equations subjected to the boundary conditions imposed at the interfaces of a multilayer stack (Fig. 2). Maxwell's equations reduce to independent sets of equations for the transverse-electric (TE) and transversemagnetic $(T M)$ polarizations. The $T E$ polarization has the electric-field vector $\boldsymbol{E}$ perpendicular to the plane of incidence, whereas it is the magnetic-field vector, $\boldsymbol{B}$, that is transverse in the $T M$ case.

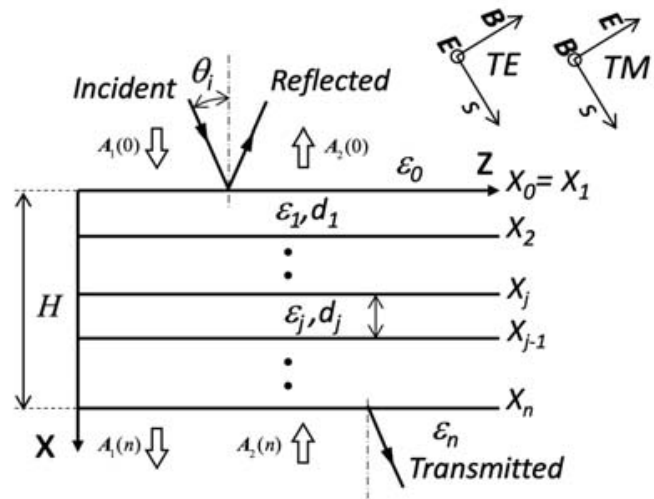

Fig. 2. N layers structure of snowpack of height $H$. Relative dielectric permittivities are, in general, complex magnitudes.
The general expression, for $T M$ polarization, of $\boldsymbol{E}$ field in a layer $j$ can be written as:

$$
\boldsymbol{E}(j)=\left[\boldsymbol{A}_{1}(j) e^{i k_{x j}\left(x-x_{j}\right)}+\boldsymbol{A}_{2}(j) e^{-i k_{x j}\left(x-x_{j}\right)}\right] e^{i\left(k_{z} z-\omega t\right)},
$$

where $\boldsymbol{A}_{1}$ represents the amplitude of a complex vector wave propagating in the positive $x$ direction (Fig. 2) and $\boldsymbol{A}_{2}$ represents a wave propagating in the negative $x$ direction. The recursive application of boundary conditions at interfaces and the propagation for $\boldsymbol{E}$ and $\boldsymbol{B}$ through the layers, conduct to a relationship between 0 and $n$ amplitudes given by:

$$
\left[\begin{array}{l}
A_{1 x}(0) \\
A_{2 x}(0)
\end{array}\right]=\left[\begin{array}{l}
M_{11} M_{12} \\
M_{21} M_{22}
\end{array}\right]\left[\begin{array}{l}
A_{1 x}(n) \\
A_{2 x}(n)
\end{array}\right]=\mathbf{M}_{T M}\left[\begin{array}{l}
A_{1 x}(n) \\
A_{2 x}(n)
\end{array}\right] .
$$

Similarly, for TE polarization,

$$
\left[\begin{array}{l}
A_{1 y}(0) \\
A_{2 y}(0)
\end{array}\right]=\mathbf{M}_{T E}\left[\begin{array}{l}
A_{1 y}(n) \\
A_{2 y}(n)
\end{array}\right] \text {. }
$$

\section{B. Reflectance Calculation for the Snowpack Multilayer}

According to the formulation outlined before, the complex reflectance, $R$, of the snowpack, assuming the condition that there is no upward propagating wave in medium $n$, i.e., $\boldsymbol{A}_{2}(n)=0$, is given, in $T E$ case, by:

$$
\left[\begin{array}{l}
A_{1 y}(0) \\
A_{2 y}(0)
\end{array}\right]=\mathbf{M}_{T E}\left[\begin{array}{c}
A_{1 y}(n) \\
0
\end{array}\right] \Rightarrow R=\frac{A_{2 y}(0)}{A_{1 y}(0)}=\left.\frac{M_{21}}{M_{11}}\right|_{T E} .
$$

Similarly, the reflectance for $T M$ case is obtained from $M_{12}$ and $M_{11}$ elements of matrix $\mathbf{M}_{T M}$.

\section{The Complex Dielectric Constant of Dry and Wet Snow}

In order to simulate the reflectance of a multilayer structure, a complex relative dielectric permittivity model for dry and wet snow in the $150 \mathrm{MHz}$ to $6 \mathrm{GHz}$ band is needed. The empirical formula from Sihvola and Tiuri is used [15].

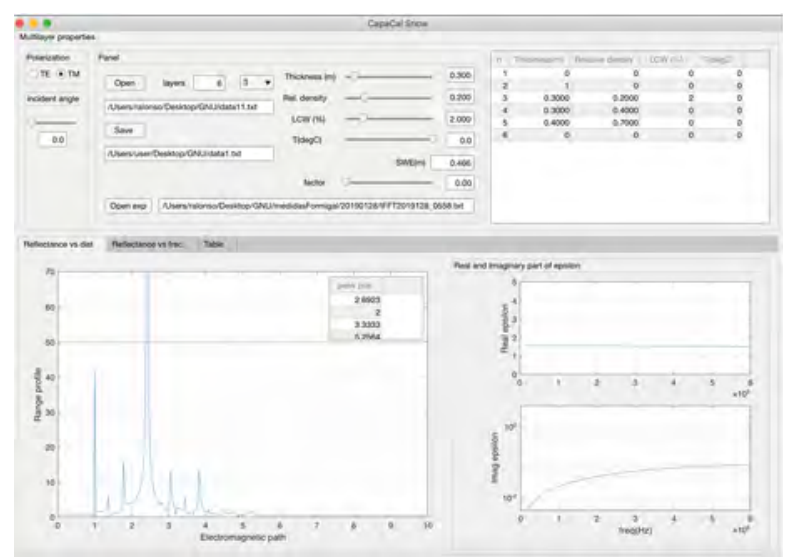

Fig. 3. Software developed to calculate the time signal of radar for a snowpack including the density, wetness and frequency of all layers.

Matrix calculations to simulate the spectral reflected signal and the IFFT vs electrical distance were graphically 
implemented in an interactive software. Measured traces can be imported in the application for comparison.

\section{Equivalent Layer of Snowpack and SWE calculation}

Using the empirical expression proposed in [15], we can observe that the real part of the square root of the relative dielectric permittivity $(\varepsilon)$ is approximately linear with snow density as shown in Fig. 4., i.e.,

$$
\operatorname{Re}(\sqrt{\varepsilon}) \simeq 1+a \rho
$$

where Re means the real part, and $\rho$ the snow layer density. This behavior is exact to dry snow and approximate for low liquid water content (LCW) snow.

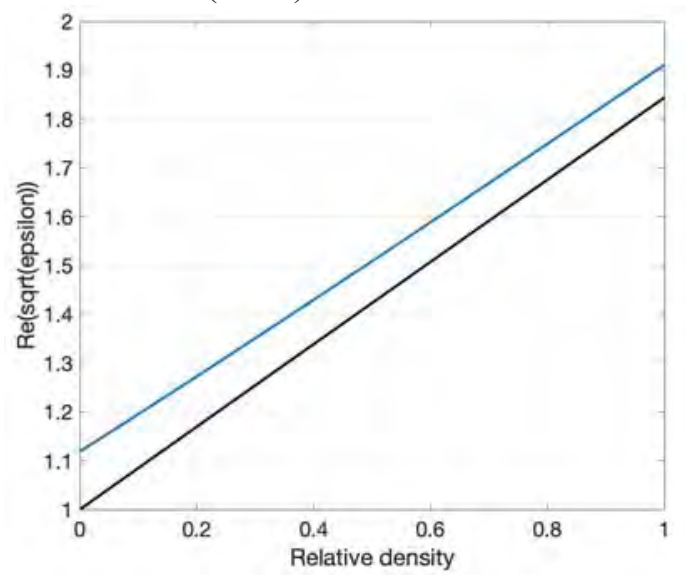

Fig. 4. Real part of the square root of the relative dielectric permittivity vs snow density at a frequency of $5 \mathrm{GHz}$. (black line $=0 \% \mathrm{LWC}$, blue line $=3 \%$. LWC).

The relation (7) enables us to relate the "fly time" or "optical/electrical path", $D$, with SWE. In fact, the optical path of a multilayer snowpack (see Fig. 2), with total thickness $H$, can be calculated, using expression (7), as follows:

$$
D=\sum_{i=1}^{n} d_{i} \operatorname{Re}\left(\sqrt{\varepsilon_{i}}\right)=H+a \sum_{i=1}^{n} d_{i} \rho_{i}=H+a S W E .
$$

We can substitute the real multilayer by one single layer of height $H$, and the same $D$ by adjusting density, $\rho_{e q}$, i.e.,

$$
D=H\left(1+a \rho_{e q}\right)=H+a H \rho_{e q} .
$$

By comparison with equation 8, we conclude that $S W E=\rho_{e q} H$. Once the thickness and the "optical path" of the snow cover are known, we can obtain SWE. The SWE of a snowpack is intrinsically related with its optical and geometrical thickness, as it was proposed by some authors [16].

\section{EXPERIMENTAL RESULTS}

\section{A. Test Site Description}

The experimental validation of our SFCW radar was carried out at the Formigal-Sarrios test site located in the Spanish Pyrenees (42 $\left.45^{\prime} 40.6^{\prime \prime} \mathrm{N} 0^{\circ} 23^{\prime} 31.8^{\prime \prime} \mathrm{W}\right)$ at an elevation of $1800 \mathrm{~m}$ a.s.l. The site was the Spanish location established by the Spanish State Meteorological Agency (AEMET) in the World Meteorological Organization Solid Precipitation Intercomparison Experiment (WMO-SPICE) [16]. Currently, the site is equipped with sensors to continuously record meteorological and snow properties. Our results have been compared with the CRN attenuation SWE measurement system from the Ebro basin hydrological service (snow gauge \#N014 of CHE), located 10m away from the SFCW radar measurement area.

\section{B. Field Measurement Radar Setup}

The SFCW radar has been implemented using a SDR platform. Nowadays, SDR is one of the emerging areas of research in low cost and accurate radars. The RF oscillator, the Rx ultrawide band amplifier, the I/Q demodulator and the ADC converters were programmed in low level software in a high-performance SDR USRP X300 with a daughterboard UBX $10-6000 \mathrm{MHz}$ from Ettus Research. The final system is a coherent and full-duplex wideband transceiver that covers frequencies from $10 \mathrm{MHz}$ to $6 \mathrm{GHz}$. Signals are low-pass filtered and FFT transformed in an industrial computer. Data are requested, to this remote system, by a $4 \mathrm{G}$ link. Finally, the system uses transmitter and receiver aluminum Vivaldi antennas (UWB3 from RFSpace) placed next to each other and protected with two radomes. Both antennas also include a thermal stabilization system to avoid undesirable freezing problems. Finally, an aluminum sheet of $1.5 \times 1,5 \mathrm{~m}$ (see Fig. 5) was placed on the ground in orthogonal direction to the antenna's axis. This sheet is the n-layer of the stack with an intrinsically imaginary behavior of $\varepsilon$. This sheet is buried underneath the snowpack during the entire snow-covered period. Table 1 summarizes the main parameters of the radar.

TABLE I. SFCW RADAR DESIGN SPECIFICATIONS

\begin{tabular}{|l|l|}
\hline Parameters & Values \\
\hline Start frequency & $150 \mathrm{MHz}$ \\
\hline Final frequency & $6 \mathrm{GHz}$ \\
\hline Step frequency, $\delta f$ & $15 \mathrm{MHz}$ \\
\hline Number of steps & 390 \\
\hline Chirp period & $\approx 80 \mathrm{~s}$ \\
\hline Maximum unambiguous range $\left(R_{u}\right)$ & $10 \mathrm{~m}$ \\
\hline Resolution (in vacuum) & $\approx 2.5 \mathrm{~cm}$ \\
\hline Intermediate frequency (IF) & $31.250 \mathrm{kHz}$ \\
\hline Sampling rate & $3.125 \mathrm{MS} / \mathrm{s}$ \\
\hline Tx and Rx antennas wide band & $675 \mathrm{MHz}-12 \mathrm{GHz}$ \\
\hline Directivity of antennas & $11 \mathrm{dBi} @ 2.4 \mathrm{GHz}$ \\
\hline Field of View of Tx and Rx system & $<15^{\circ}$ \\
\hline
\end{tabular}

Fig. 5 shows a scheme of the system. For calibration purposes, a temporal metallic plate is placed at $\mathrm{x}=0$ position and is used to optimize the receiver gain and the phase measurement in each frequency of the chirp. The file 
generated represents the reference for the succeeding measurements.

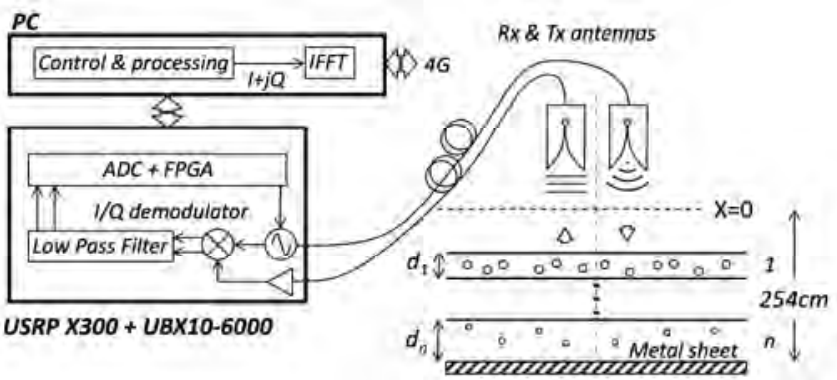

Fig. 5. Blocks of the radar system. The main operations of radar are implemented by software in the USRP X300 module. The distance from "virtual" 0 to metal sheet is $254 \mathrm{~cm}$.
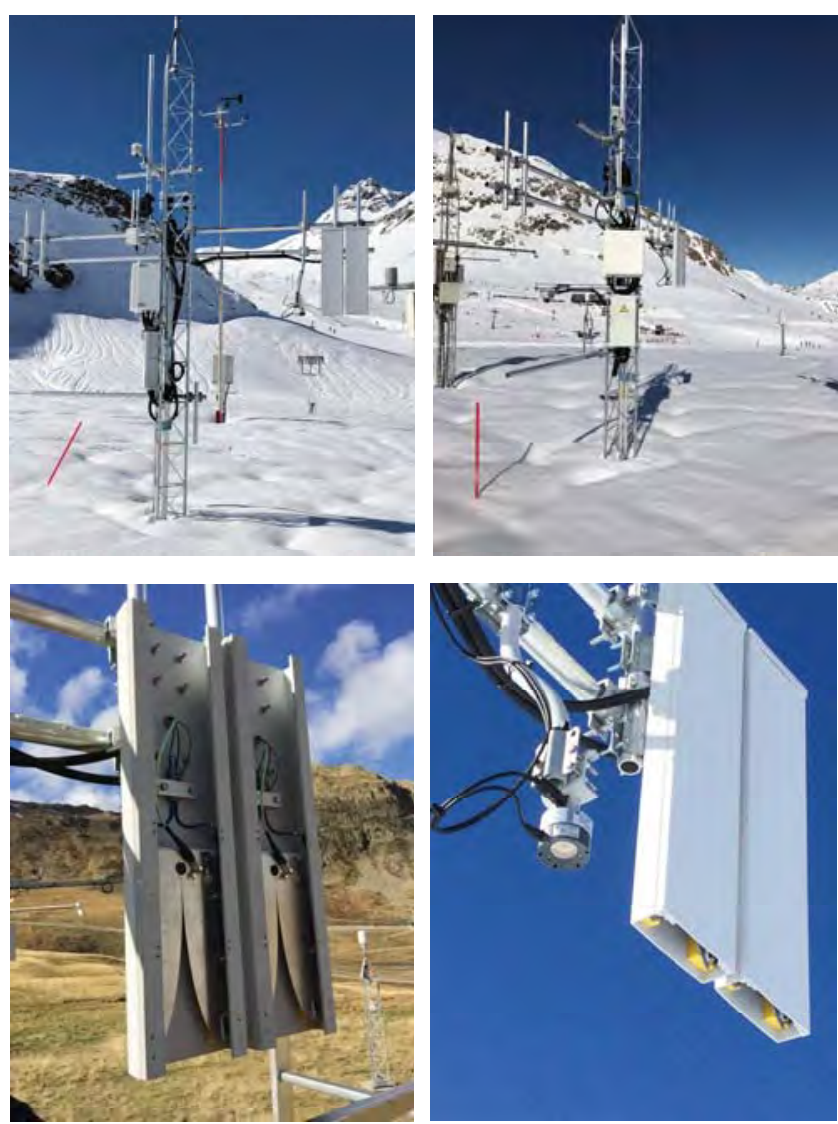

Fig. 6. Upper images show the $6 \mathrm{~m}$ tower with all the elements of the system. Bottom images show detail of the Vivaldi antennas protected by radomes. An acoustic sensor (Campbell SR50AH) was included to validate heigth measurements from the radar.

\section{Measurement process}

On 25 January 2019, before the first important snowfall of 2019, the SFCW radar was operative. A series of measurements of the distance of metal sheet $(254 \mathrm{~cm})$ was made with the radar, in order to obtain later the height of the snow cover.

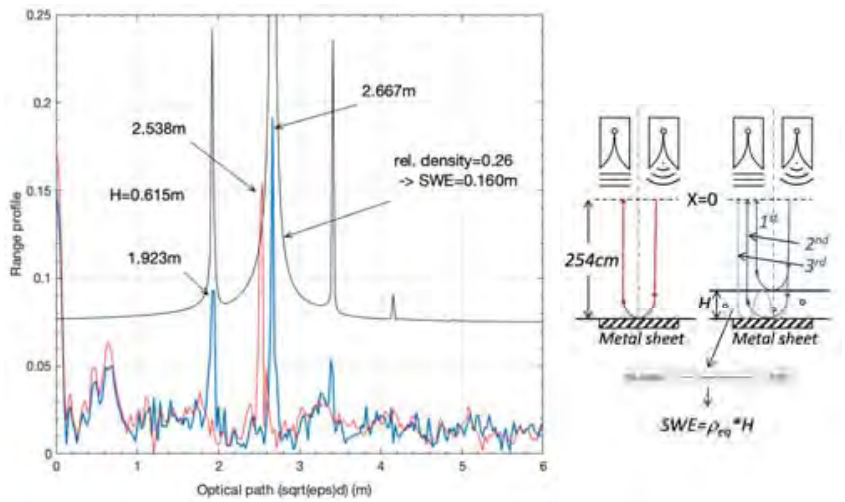

Fig. 7. Measurement example of SWE. The $3^{\text {rd }}$ blue peak represents a doble reflection in snow layer.

To understand the SWE measurement, we show the process in Fig. 7. The red trace shows the reflectance of the metal sheet without snow cover, and the blue one, the measurement after snowfall. The snow cover height, $H$, is the distance between the $1^{\text {st }}$ blue peak and the red peak. The optical depth, $D$, is the distance between the $1^{\text {st }}$ and $2^{\text {nd }}$ blue peaks. The black line is the simulated signal of a single layer obtained by adjusting the density $\left(\rho_{e q}\right)$ slider of a snow layer of height $H$ up to $D$ optical path. The $S W E$ is $\rho_{e q} H$. In Fig. 8 we compare measurements of SWE with our radar and with the CRN gauge located next to the radar. Also $H$ measurement from our radar system is shown. As can be seen, there is a reasonable agreement between them.

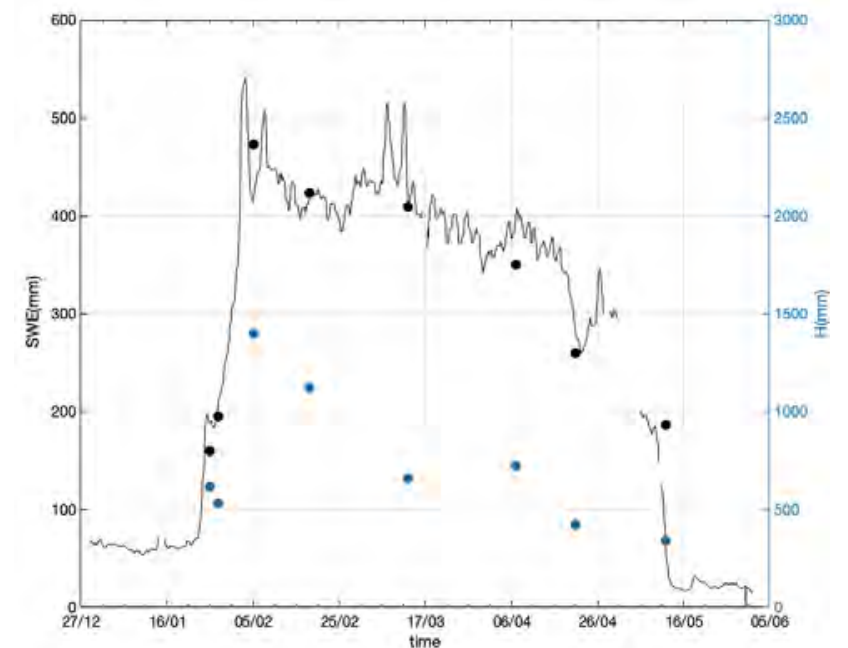

Fig. 8. Validation of the radar SFCW tecnique. SWE data from CRN gauge are represented in black continous line. Black and blue dots are SWE and thickness of snowpack estimated by SFCW radar, respectively.

\section{CONCLUSIONS AND FUTURE WORK}

The main conclusion of this work is the validation of the proposed SFCW radar technique and the electromagnetic model of the snowpack to obtain SWE for hydrological purposes. The agreement with the cosmic ray technique is reasonable. Future work must continue with a precise estimation of error by comparison with gravimetric measurements of SWE. Further improvements of SFCW 
hardware and software are necessary to increase SNR ratio, "ghost" peaks detection algorithms and bandwidth increase of frequency chirp to increase resolution in order to measure snowpack stratigraphy.

\section{ACKNOWLEDGMENTS}

This work was partly supported by DGA-FSE under grant T20_17R to the Photonics Technologies Group of University of Zaragoza. We would like to thank everyone who helped us in this project, especially to those at the Infrastructure Division of the Aragon Regional Office of AEMET and at the Ebro basin hydrological service (CHE), for allowing free use of their facilities, for their support and the good advice given during the installation of our radar system. Finally, to Formigal Ski Resort for the facilities offered.

\section{REFERENCES}

[1] N. J. Kinar and J. W. Pomeroy, "Measurement of the physical properties of the snowpack," Rev. Geophys., vol. 53, pp. 481-544, Jun 2015.

[2] E. Paquet, M. Laval, L.M. Basalaev, A. Belov, E. Eroshenko, V. Kartyshov, A. Struminsky, and V.L. Yanke, "An Application of Cosmic-Ray Neutron Measurements to the Determination of theSnow Water Equivalent," Proceedings of the 30th International Cosmic Ray Conference, vol. 1, pp. 761-764, 2008

[3] M. Kodama, K. Nakai, S. Kawasaki, and M. Wada, "An application of cosmic-ray neutron measurements to the determination of the snowwater equivalent,” J. Hydrol., vol. 41, pp. 85-92, Aug 1979.

[4] N.J. Kinar, and J.W. Pomeroy, "Automated Determination of Snow Water Equivalent by Acoustic Reflectometry," IEEE Transactions on Geoscience and Remote Sensing, vol. 47, pp. 3161-3167, Sep 2009.

[5] A. Lundberg, H. Thunehed, and J. Bergström "Impulse Radar Snow Surveys -Influence of Snow Density," Nordic Hydrology, vol. 31, pp. $1-14,2000$.

[6] A. Lundberg, and H. Thunehed, "Snow Wetge Influence on Impulse Radar Snow Surveys. Theoretical and Laboratory study," Nordic Hydrology, vol. 31, pp. 89-106, 2000

[7] H.P. Marshall, and G. Koh, "FMCW radars for snow research," Cold Regions Science and Technology, vol. 52, pp. 118-131, Apr 2008

[8] R. Okorn, G. Brunnhofer, T. Platzer, A. Heilig, L. Schmid, C. Mitterer, J. Schweizer, and O. Eisen, "Upward-looking L-band FMCW radar for snow cover monitoring," Cold Regions Science and Technology, vol. 103, pp. 31-40, Jul 2014.

[9] M. Pasian, M. Barbolini, F. Dell'Acqua, P.F. Espín-López, and L. Silvestri, "Snowpack Monitoring Using a Dual-Receiver Radar Architecture," IEEE Transactions on Geoscience and Remote Sensing, vol. 57, pp. 1195-1204, Feb 2019.

[10] N. Yankieluna, W. Rosenthalb, and R.E. Davis, "Alpine snow depth measurements from aerial FMCW radar," Cold Regions Science and Technology., vol. 40, pp. 123-134, Jun 2004.

[11] C. Nguyen, and J. Park, Stepped-Frequency Radar Sensors. Theory, Analysis and Design, Springer International Publishing, ISBN: 978-3319-12271-7, 2016.

[12] H. Cory, and C. Zach, "Wave propagation in metamaterial multilayered Structures," Microwave and Optical Technology Letters, vol. 40, pp. 460-465, 2004.

[13] K. Kurosawa, R.M. Pierce, S. Ushioda, and J.C. Hemminger, "Ramanscattering and attenuated-total-reflection studies of surface-plasmon polaritons," Physical Review B, vol. 33, pp. 789-798, Jan 1986.

[14] M. Tiuri, A. Sihvola, E. Nyfors, and M. Hallikainen, "The complex dielectric constant of snow at microwave frequencies," IEEE Journal of Oceanic Engineering, Vol. 9, pp. 377-382, 1984.

[15] S.T. Buisán, M.E. Earle, J. L. Collado, J. Kochendorfer, J. Alastrué, M. Wolff, C.D. Smith, and J.I. López-Moreno, "Assessment of snowfall accumulation underestimation by tipping bucket gauges in the Spanish operational network," Atmospheric Measurement Techniques, Vol. 10, pp. 1079-1091, March 2017.

[16] A. Lundberg, H. Thunehed, and J Bergström, "Impulse radar snow surveys-influence of snow," Hydrology Research, Vol. 31, pp. 1-14, Apr. 2000. 